\title{
THEORETICAL INVESTIGATION OF $\psi$-SPLITTING AFTER PLASTIC DEFORMATION OF TWO-PHASE MATERIALS
}

\author{
M. Berveiller ${ }^{(a)}$ J. Krier $^{(b)}$, H. Ruppersberg ${ }^{(b)}$, C.N.J. Wagner ${ }^{(c)}$ \\ (a) L.P.M.M.-I.S.G.M.P., Metz, France \\ (b) Ingenieurwissenschaften Universitat Saarbrücken, W-Germany \\ (c) Materials Science and Engineering Department, UCLA, Los Angeles, USA
}

\section{INTRODUCTION}

X-ray stress analysis is based on the $\sin ^{2} \psi$ - law which is described in more detail in a paper by Krier et al. [1] published in these proceedings. For the present discus sions, we need the more complete equation relating the elastic strain $\varepsilon_{\varphi \psi}$ with all tensor components $\sigma_{\mathrm{ij}}$ of the stress, including the $\sigma_{3 j}$-terms :

$$
\begin{aligned}
\varepsilon_{\varphi \psi}= & \frac{1}{2} S_{2}\left[\sigma_{11} \cos ^{2} \varphi+\sigma_{22} \sin ^{2} \varphi+\sigma_{12} \sin 2 \varphi-\sigma_{33}\right] \sin ^{2} \psi+S_{1}\left[\sigma_{11}+\sigma_{22}+\sigma_{33}\right] \\
& +\frac{1}{2} S_{2}\left[\sigma_{33}+\sigma_{13} \cos \varphi \sin 2 \psi+\sigma_{23} \sin \varphi \sin 2 \psi\right]
\end{aligned}
$$

$\varepsilon_{\varphi \psi}$ is the average normal strain of the reflecting grains pointing in the $\mathrm{N}^{\mathrm{h}}$ direc tion. $N^{\mathrm{h}}$ is perpendicular to the reflecting planes. $\sigma_{\mathrm{ij}}$ corresponds to the mean stress ob tained by averaging over the total volume of the reflecting phase. $S_{1}$ and $S_{2}$ are the $\mathrm{X}$-ray elastic constants. The subscripts of $\sigma$ and $\varepsilon$ refer to the coordinates of the speci men. The 3-direction $\mathrm{P}^{3}$ is defined to be perpendicular to the flat surface which is in vestigated with the $X$-rays. $\mathrm{N}^{\mathrm{h}}$ then has the components $\mathrm{N}_{1}^{\mathrm{h}}(\sin \psi \cos \varphi), \mathrm{N}_{2}^{\mathrm{h}}(\sin \psi$ $\sin \varphi)$ and $\mathrm{N}_{3}^{\mathrm{h}}(\cos \psi) . \psi$ and $\varphi$ are the angles between $\mathrm{N}^{\mathrm{h}}$ and $\mathrm{P}^{3}$ and between $\mathrm{P}^{1}$ and the projection of $N^{h}$ on the 1-2 plane, respectively. The equation (1) is valid for macroscopic isotropic elasticity of the diffracting phase.

$\sigma_{13}$ and/or $\sigma_{23}$ terms occur if the principal axes of $\sigma$ are tilted with respect to $\mathrm{P}^{3}$. Be cause these terms are multiplied in equation (1) by $\sin 2 \psi, \varepsilon_{\varphi \psi}$ will be different from $\varepsilon_{\varphi-\psi}$. This effect is called " $\psi$-splitting" and may be observed after machining (milling, turning, grinding...) or directional shot peening of metals. With few exceptions, $\psi$-splitting was observed only for multiphase materials. It is strongest in the plane which contains $\mathrm{P}^{3}$ and the direction of machining or shot peening and it is smaller or even disappears in the plane perpendicular to this direction. More details are given in the references [2] [3] [4].

In principle, $\psi$-splitting may be related to very different origins namely (i) special tex ture of the surface layer, (ii) surface roughness and/or (iii) complex stress fields which yield a tilting of the principal axes of the average stress and strain tensors with respect to $\mathrm{P}^{3}$. 
We study only the last mentioned case for which eq.1 applies and which seems to be the most frequent one. We start from a micromechanical model which is based on the plastic inclusion problem of a material containing a hard and a ductile phase. We neglect surface effects and absorption of the $\mathrm{X}$-rays.

\section{THEORY}

We consider a material which is homogeneous on a macroscopic scale but heterogeneous on a microscopic level and composed of a ductile matrix and ellipsoïdal nonductile inclusions of volume fraction $\mathrm{f}$. The grains of both phases have the same isotropic elastic behaviour described by the shear modulus $\mu$ and Poisson's ratio $v$.

This material undergoes a global deformation $E^{t}$ composed of an elastic deformation $E^{e}$ and a plastic part $\mathrm{E}^{\mathrm{P}}$. Describing by $\varepsilon^{\mathrm{pM}}$ and $\varepsilon^{\mathrm{pE}}(=0)$ the mean plastic strain in the matrix $(\mathrm{M})$ and the inclusions $(\mathrm{E})$ respectively, one can write

$$
E_{i j}^{p}=f \varepsilon_{i j}^{p E}+(1-f) \varepsilon_{i j}^{p M}=(1-f) \varepsilon_{i j}^{p M}
$$

The solution of the problem of an inclusion in a matrix (Eshelby [5], Kröner [6]) al lows us to write, using the Einstein convention

$$
E_{i j}^{t}-\varepsilon_{i j}^{E}=S_{i j k l}\left(E_{k l}^{p}-\varepsilon_{k l}^{p}\right)
$$

If $\mathrm{E}^{\mathrm{e}}=0$ (no applied elastic strains) and since $\varepsilon^{\mathrm{pE}}=0$, equation (3) can be written as

$$
\varepsilon_{\mathrm{ij}}^{\mathrm{eE}}=\left(\mathrm{I}_{\mathrm{ijkl}}-S_{\mathrm{ijkl}}\right) \mathrm{E}_{\mathrm{kl}}^{\mathrm{p}}
$$

where $I_{i j k l}$ is the identity tensor and $S_{i j k l}$ is the Eshelby tensor. The normal average strain of the inclusions becomes for the $\mathrm{N}^{\mathrm{h}}$ direction

$$
\varepsilon_{\varphi \psi}^{E}=N_{i}^{h} N_{j}^{h}\left(I_{i j k l}-S_{i j k k}\right) E_{k l}^{p}
$$

The corresponding value of $\varepsilon^{M} \varphi \psi$ is obtained from the equilibrium conditions for $E^{e}=$ 0 , i.e. :

$$
\varepsilon_{\varphi \psi}^{\mathrm{M}}=-\frac{\mathrm{f}}{1-\mathrm{f}} \varepsilon_{\varphi \psi}^{\mathrm{E}}
$$

Equation (5) indicates that there are two sources for $\psi$-splitting namely anisotropic $S$ or $\mathrm{E}^{\mathrm{P}}$ tensors with tilted principal axes with respect to $\mathrm{P}^{3}$. We [7] recently studied the last mentioned case which is observed for spherical inclusions and which yields an isotropic S-tensor of the form [5] 
$S_{i j k l}=\frac{1}{3}(\alpha-\beta) \delta_{i j} \delta_{k l}+\frac{1}{2} \beta\left(\delta_{i k} \delta_{j 1}+\delta_{i 1} \delta_{j k}\right) \quad$ with $\alpha=\frac{1}{3} \frac{1+v}{1-v}$ and $\beta=\frac{2}{15} \frac{4-5 v}{1-v}$

In the special case of $\mathrm{E}^{\mathrm{p}}$ corresponding to oblique shot peening

$$
E_{11}^{p}=E_{22}^{p}=-\frac{1}{2} E_{33}^{p}, E_{13}^{p}=E_{31}^{p}, \text { other } E_{i j}^{p}=0
$$

we obtained the following equation for $\varepsilon^{M} \varphi$

$$
\varepsilon_{\varphi \psi}^{\mathrm{M}}=-\frac{1}{2} \frac{\mathrm{f}}{1-\mathrm{f}} \mathrm{E}_{33}^{\mathrm{p}}\left\{1-\frac{3}{2} \sin ^{2} \psi+\frac{\mathrm{E}_{13}^{\mathrm{p}}}{\mathrm{E}_{33}^{\mathrm{p}}} \sin 2 \psi \cos \varphi\right\}
$$

In fig. 1 are given several $\varepsilon^{\mathrm{M}} \varphi \psi / \mathrm{E}^{\mathrm{p}} 33$ vs. $\sin ^{2} \psi$ curves which have been calculated for different volume fractions $\mathrm{f}$ and for $\mathrm{E}^{\mathrm{p}}{ }_{13} / \mathrm{E}^{\mathrm{P}} 33=0.3$. The general behaviour corres ponds well to experimental observations [2] [3] [4].

In the next section we study the case of parallel non-spherical inclusions. The corresponding anisotropic S-tensor yields $\psi$-splitting if it is tilted with respect to $\mathrm{P}^{3}$ even if a principal axis of $\mathrm{E}^{\mathrm{P}}$ coincides with $\mathrm{P}^{3}$. Practical examples would be lamellar eutectics or composite materials with parallel reinforcements.

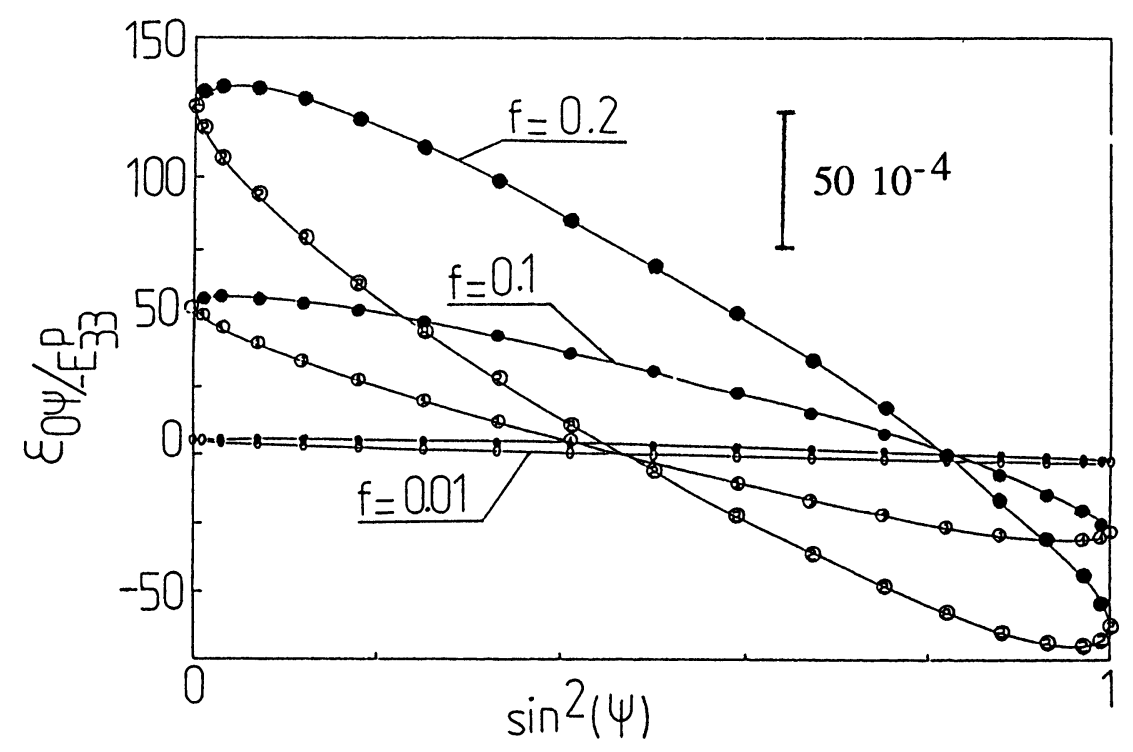

Fig $1 \varepsilon^{\mathrm{M}}{ }_{0 \psi / E^{\mathrm{p}}} \mathrm{p}_{33}$ vs. $\sin ^{2} \psi$ curves of the matrix for positive (o) and negative ( $\left.\bullet\right) \psi$ values. A value of 0.3 was chosen for $E^{\mathrm{P}} 13 / \mathrm{E}^{\mathrm{P}} 33$. The curves are calculated for the volume fractions $f$ given in the the figure [7]. 


\section{APPLICATIONS AND DISCUSSION}

We investigate penny-shape ellipsoïdal inclusions with axes $\mathrm{a}=\mathrm{b}$ and $\mathrm{c} / \mathrm{a}<<1$.

If $S$ is referred to these axes, it has only the following non-vanishing components [8] :

$$
\begin{aligned}
& S_{1111}=S_{202}=\frac{13-8 v}{32(1-v)} \pi \frac{c}{a}, S_{3333}=1-\frac{1-2 v}{1-v} \pi \frac{c}{a}, S_{3311}=S_{332}=\frac{v}{1-v}\left(1-\frac{4 v-1}{8 v} \pi \frac{c}{a}\right) \\
& S_{1122}=S_{2211}=\frac{8 v-1}{32(1-v)} \pi \frac{c}{a}, S_{1133}=S_{2233}=\frac{2 v-1}{8(1-v)} \pi \frac{c}{a}, S_{1313}=S_{2323}=\frac{1}{2}\left(1+\frac{v-2}{1-v} \frac{\pi}{4} \frac{c}{a}\right) \\
& S_{1212}=\frac{7-8 v}{32(1-v)} \pi \frac{c}{a}
\end{aligned}
$$

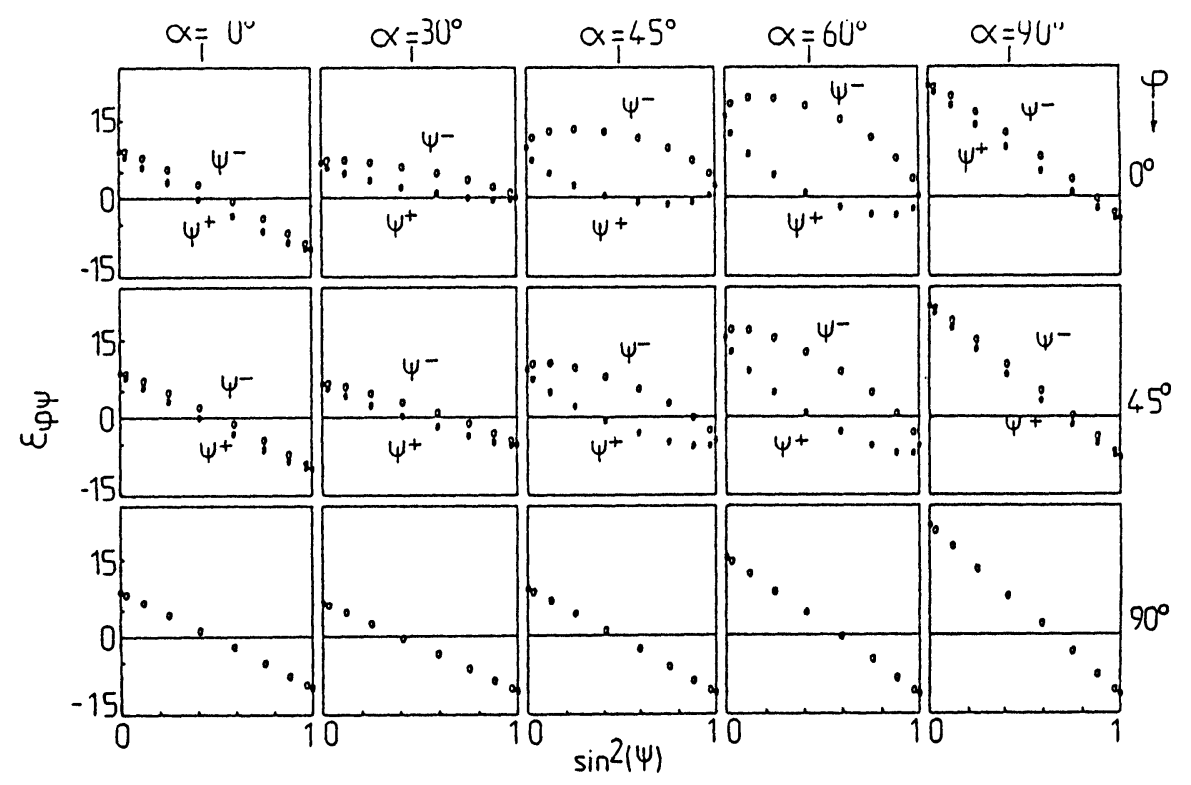

Fig $2 \varepsilon^{\mathrm{M}} \varphi \psi^{/ E^{\mathrm{P}}} 33$ vs. $\sin ^{2} \psi$ curves of the ductile matrix with penny-shape non duc tile inclusions after plastic deformation corresponding to perpendicular shot peening. The curves are given for positive ( $\bullet$ ) and negative (o) $\psi$ angles, for $\varphi=0^{\circ}, 45^{\circ}, 90^{\circ}$ and for 5 different orientations of the inclusions with respect to the surface, ranging from $\alpha=0^{\circ}$ (parallel) to $\alpha=90^{\circ}$ (perpendicular).

The orientation of the inclusions with respect to the P-coordinates is described analogous to $\mathrm{N}^{\mathrm{h}}$ by the angles $\alpha$ between $\mathrm{P}^{3}$ and $\mathrm{c}$, corresponding to $\psi$, and $\gamma$ corresponding to $\varphi$. We first discuss perpendicular shot peening, which yields the plastic strain 
$E_{11}^{p}=E_{2}^{p}=-E_{33}^{p} / 2 \quad$ other $E_{i j}^{p}=0$

and the normal elastic strain of the matrix in the $\mathrm{N}^{\mathrm{h}}$-direction

$$
\varepsilon_{\varphi \psi}^{M}=-\frac{f}{1-f} \frac{E_{3}^{P}}{2}\left(A+B \sin ^{2} \psi+C \sin \psi \cos \psi\right)
$$

with, for $\gamma=\varphi=0$.

$$
\begin{aligned}
& A=\left(3+\frac{v}{1-v}-\frac{45}{32(1-v)} \pi \frac{c}{a}\right) \cos ^{4} \alpha+\left(5+\frac{v}{1-v}-\frac{87-48 v}{16(1-v)} \pi \frac{c}{a}\right) \cos ^{2} \alpha-2+\frac{31-32 v}{32(1+v)} \pi \frac{c}{a} \\
& B=6\left(1+\frac{v}{1-v}-\frac{45}{32(1-v)} \pi \frac{c}{a}\right) \cos ^{4} \alpha-\left(7+\frac{5 v}{1-v} \cdot \frac{285-48 v}{32(1-v)} \pi \frac{c}{a}\right) \cos ^{2} \alpha+2+\frac{v}{1-v} \cdot \frac{27}{32(1-v)} \pi \frac{c}{a} \\
& C=2 \cos \alpha \sin \alpha\left\{2+\frac{v}{1-v} \cdot \frac{75-24 v}{32(1-v)} \pi \frac{c}{a}-3\left(1+\frac{v}{1-v}-\frac{45}{32(1-v)} \pi \frac{c}{a}\right) \cos ^{2} \alpha\right\}
\end{aligned}
$$

The term which is proportional to $C$ yields the $\psi$-splitting seen in Fig. 2 and which depends on $\alpha$ and $c / a$. The curves are given for $\gamma=0, f=0.2, c / a=0.1$ and $E^{P}{ }_{33}=0.1$.

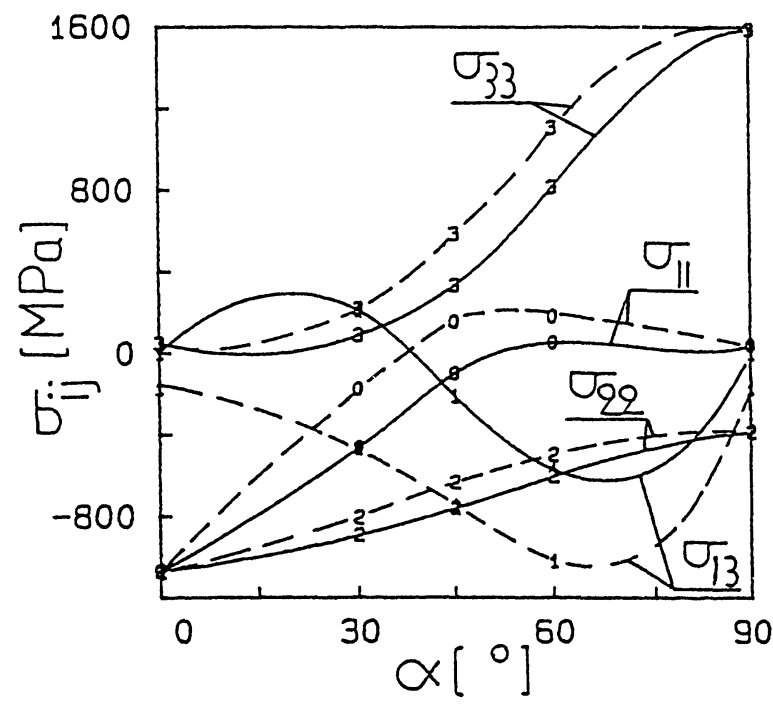

Fig 3 Stress components $\sigma^{\mathrm{M}}$ ij observed after plastic deformation of a ductile matrix $M$ with penny-shape inclusions. The curves are plotted against the angle $\alpha$ between the normal on the specimen and the normal on the inclusion $(\gamma=0)$.

Full curves $: \sigma$ values after normal shot peening

Dashed curves : $\sigma$ values after oblique shot peening 
Equation (1) makes a more condensed description of the $\varepsilon_{\varphi \psi}$ curves in Fig. 2 possible by just plotting the corresponding non vanishing $\sigma_{\mathrm{ij}}$ components vs. $\alpha$. This is done in Fig. 3 using $\mu=3.10^{4} \mathrm{MPa}$ and $v=0.3$. Remarkable are the inversion of the sign of the full drawn $\sigma_{13}$ at about $\alpha=40^{\circ}$ and the strong $\sigma_{33}$ values observed at large $\alpha$ angles. It seems evident that phase-specific average $\sigma_{33}$ stresses will also occur for specimens consisting of randomly oriented penny packages. This is a quite realistic description of lamellar eutectics and means that, e.g., $\sigma_{33}$ stresses might occur in rolled steel plates.

As a last example, we studied the combination of both $\mathrm{E}^{\mathrm{p}}$ and $\mathrm{S}$-induced splitting. $\mathrm{E}^{\mathrm{p}}$, according to equations (5) and (6), yields the dashed drawn $\sigma_{\mathrm{ij}}$-curves show in fig.3. These look similar to the previous results with the exception of $\sigma_{13}$. It is now seen to be negative over the whole $\alpha$-range.

Finally, we should point out that our calculations are based on the hypothesis of a purely elastic accomodation of the plastic incompatibilities which overestimates the internal stresses. Thus the effects shown in the figures are too strong. In reality, there will be partial plastic accomodation which yields stress gradients. A line profile analysis of the diffraction peaks might give informations about these gradients. This would be very helpful for understanding the interactions between a metallic matrix and the reinforcements of composite materials.

\section{ACKNOWLEDGEMENTS}

The financial supports of E.C. and PROCOPE are gratefully acknowledged.

\section{REFERENCES}

1. J. Krier, H. Ruppersberg, M. Berveiller and P. Lipinski, This conference

2. M. Wakabayashi, M. Nakayama and A. Nagata, J. Soc. Prec. Engng 43, 661 (1977)

3. I.C. Noyan and J.B. Cohen, Residual Stress Measurement by diffraction and interpretation, Springer Verlag (1987)

4. V. Hauk and P. Stuitje, "Eigenspannungen : Entstehung, Messung, Bewertung" DGM, 59 (1983)

5. J.D. Eshelby, Proc. Roy. Soc., A319, 247 (1970)

6. E. Kröner, Acta Met. 9, 155 (1961)

7. M. Berveiller, J. Krier, H. Ruppersberg and C.N.J. Wagner, CETIM, Journées

Nat. sur les cont. résiduelles, Senlis - (1990)

8. T. Mura, Micromechanics of defects in solids, Mart. Nijhoff Publ. (1982) 